\title{
A note on the generalized Lagally theorem
}

\section{A. BIESHEUVEL}

Technical University Twente, Department of Applied Physics, P.O. Box 217, 7500 AE Enschede, The Netherlands

(Received November 29, 1984)

\section{Summary}

A derivation of the generalized Lagally theorem for the forces on a body in translational motion in an unsteady inhomogeneous flow field of an inviscid incompressible fluid is given. It is also shown that some well-known results for the forces on spherical bodies can be obtained in a simple way by using this theorem.

\section{Introduction}

In this paper a generalization of what is known as the Lagally theorem is considered. This theorem expresses the hydrodynamic force on a body in an unsteady inhomogeneous flow of an incompressible inviscid fluid in terms of the strengths of the singularities associated with the analytically continued flow within the body. When the exterior potential field is given by

$$
\phi(x)=-\frac{1}{4 \pi} \sum_{\mathrm{s}}(-1)^{q} M_{q \mathrm{~s}} \frac{\partial^{q}}{\partial x^{q}}\left(\frac{1}{\left|x-x_{\mathrm{s}}\right|}\right),
$$

where $M_{q \mathrm{~s}}$ is the strength of a general multipole of order $q$ at the point $\boldsymbol{x}_{\mathrm{s}}$ and $\Sigma_{\mathrm{s}}$ denotes summation over all singularities, the generalized Lagally theorem yields the following expression for the hydrodynamic force on a deformable body:

$$
F=\rho_{1}\left\{\frac{\mathrm{d}}{\mathrm{d} t}\left[v_{\mathrm{c}} V-\sum_{\mathrm{s}} M_{q \mathrm{~s}} \frac{\partial^{q}}{\partial x^{q}}(\boldsymbol{x})_{\mathrm{s}}+\frac{\mathrm{d}}{\mathrm{d} t}\left(x_{\mathrm{c}} V\right)\right]-\sum_{\mathrm{s}} M_{q \mathrm{~s}} \frac{\partial^{q}}{\partial \boldsymbol{x}^{q}}\left(\boldsymbol{u}_{\mathrm{r}}\right)_{\mathrm{s}}\right\}
$$

Here $\rho_{\mathrm{l}}$ is the fluid density, $v_{\mathrm{c}}$ is the velocity of the centre $\boldsymbol{x}_{\mathrm{c}}$ of volume $V$ of the body; $\boldsymbol{u}_{\mathrm{r}}$ is the velocity induced at the location of a multipole in $x_{s}$ by all other external flow producing mechanisms and ()$_{\mathrm{s}}$ denotes that the various derivatives should be evaluated at the points $x_{s}$.

Theorems on the relations between certain kinds of added masses of bodies moving steadily through an inviscid liquid, the forces acting upon them and the singularities of the flow field were given in the 1920's by M. Lagally, G.I. Taylor and M.M. Munk, and generalizations to unsteady motion and all kinds of added masses were published in the 1950's by W.E. Cummins, G. Birkhoff, L. Landweber and C.S. Yih (references may be 
found in Landweber and Yih [1]). A recent rigorous derivation of the generalized Lagally theorem by Landweber and Miloh [2] contained some errors. Their corrected derivation (Landweber and Miloh [3]) now consists of, as they call it, "some exercises in spherical harmonics".

The purpose of this note is to give a shorter and much simpler alternative to the Landweber and Miloh derivation. It partly follows their derivation but uses at certain stages ideas put forward in van Wijngaarden [4]. In the final section some well-known results are established again as demonstrations of the use of the Lagally theorem. These somewhat obvious examples were also chosen in order to enable us to make a brief comment on the use of relations for single particles in theories for two-phase bubbly liquid flows.

\section{A derivation of the generalized Lagally theorem}

Consider a deformably body moving without rotation in an inviscid, incompressible fluid with density $\rho_{1}$. We employ a co-ordinate system fixed at infinity and denote the position vector of a point by $\boldsymbol{x}$. The centre of the body $\boldsymbol{x}_{\mathrm{c}}$ is taken to move with velocity

$$
v_{\mathrm{c}}=\frac{\mathrm{d} x_{\mathrm{c}}}{\mathrm{d} t}
$$

and in addition the body surface is allowed to have a deformation velocity $v_{\mathrm{d}}$ relative to the centre of the body. The flow is irrotational and characterized by the velocity potential $\phi$ and the velocity $u=\nabla_{x} \phi$. The hydrodynamic force exerted on the body by the surrounding fluid arises from the pressure at the body surface

$$
\boldsymbol{F}=-\int p \boldsymbol{n d} A
$$

and with the aid of Bernoulli's theorem, we have

$$
F=\rho_{1} \int \frac{\partial \phi}{\partial t} n \mathrm{~d} A+\rho_{1} \int \frac{1}{2} u \cdot u n \mathrm{~d} A-\rho_{1} \int g \cdot x \boldsymbol{n d} A
$$

The last integral in (2) represents the buoyancy force on the body and will be omitted in what follows.

The expression (2) may also be written as

$$
\boldsymbol{F}=\rho_{1} \frac{\mathrm{d}}{\mathrm{d} t} \int \phi n \mathrm{~d} A+\rho_{1} \int\left(\frac{1}{2} \boldsymbol{u} \cdot \boldsymbol{u n}-\boldsymbol{u} \cdot \boldsymbol{v}_{\mathrm{c}} \boldsymbol{n}-\boldsymbol{u} v_{\mathrm{d}} \cdot \boldsymbol{n}\right) \mathrm{d} A,
$$

where $\mathrm{d} / \mathrm{d} t$ is the material derivative following the motion of the body. The first term on the right-hand side may be called the acceleration reaction $\left(\boldsymbol{F}_{\mathrm{A}}\right)$, while the second term may be referred to as the steady force $\left(\boldsymbol{F}_{\mathrm{s}}\right)$, since it represents the force on the body in steady motion. As mentioned in Section 1 , the Lagally theorem expresses these forces in terms of products of the singularity strengths and gradients of the velocity potential to be evaluated at the location of the singularities. To obtain these expressions we consider the acceleration reaction first, postponing the treatment of the steady force at this moment. 


\subsection{The acceleration reaction}

Apply Green's reciprocal theorem to the volume bounded externally by the body surface and internally by surfaces $A_{\epsilon_{s}}$ enclosing small regions $V_{\mathrm{e}_{\mathrm{s}}}$ about the points $\boldsymbol{x}_{\mathrm{s}}$, at which the singularities are located. This yields

$$
F_{\mathrm{A}}=\rho_{1} \frac{\mathrm{d}}{\mathrm{d} t}\left[\int \boldsymbol{x} \nabla_{\boldsymbol{x}} \phi \cdot n \mathrm{~d} A+\sum_{\mathrm{s}} \int\left(\phi n-x \nabla_{x} \phi \cdot n\right) \mathrm{d} A_{\epsilon_{\mathrm{s}}}\right] .
$$

With the aid of Gauss' theorem we obtain for the first integral

$$
\int x \nabla_{x} \phi \cdot n \mathrm{~d} A=\int x v_{\mathrm{c}} \cdot n \mathrm{~d} A+\int x v_{\mathrm{d}} \cdot n \mathrm{~d} A=v_{\mathrm{c}} V+\int x v_{\mathrm{d}} \cdot n \mathrm{~d} A,
$$

where $V$ is the volume of the body. Further, by the Reynolds transport theorem,

$$
\int x v_{\mathrm{d}} \cdot n \mathrm{~d} A=\frac{\mathrm{d}}{\mathrm{d} t}\left(x_{\mathrm{c}} \mathcal{K}\right)
$$

At each point $\boldsymbol{x}_{\mathrm{s}}$ the potential and the velocity can be taken to consist of a singular part $\left(\phi_{\mathrm{s}}, \boldsymbol{u}_{\mathrm{s}}\right)$, where the singularities are assumed to be multipoles of order $q$ and strength $M_{q \mathrm{~s}}$,

$$
\phi_{\mathrm{s}}(x)=-\frac{M_{q \mathrm{~s}}}{4 \pi} \frac{\partial^{q}}{\partial x_{\mathrm{s}}^{q}}\left(\frac{1}{\left|x-x_{\mathrm{s}}\right|}\right), \quad u_{\mathrm{s}}(x)=-\frac{M_{q \mathrm{~s}}}{4 \pi} \frac{\partial^{q}}{\partial x_{\mathrm{s}}^{q}}\left(\nabla_{x} \frac{1}{\left|x-x_{\mathrm{s}}\right|}\right),
$$

and a regular part $\left(\phi_{\mathrm{r}}, \boldsymbol{u}_{\mathrm{r}}\right)$ associated with other mechanisms producing the flow at $\boldsymbol{x}_{\mathrm{s}}$. Since

$$
\lim _{A_{\mathrm{e}_{\mathrm{s}} \rightarrow 0}} \int\left(\phi_{\mathrm{r}} n-x \nabla_{x} \phi_{\mathrm{r}} \cdot \boldsymbol{n}\right) \mathrm{d} A_{\epsilon_{\mathrm{s}}}=0
$$

the regular parts of the potentials do not contribute to the second integral in (3).

To obtain the contributions due to the singular parts, first consider the singularities to consist only of monopoles. We then have

$$
\sum_{\mathrm{s}} \int\left(-\frac{m_{\mathrm{s}}}{4 \pi\left|\boldsymbol{x}-\boldsymbol{x}_{\mathrm{s}}\right|} \boldsymbol{n}-\boldsymbol{x} \frac{m_{\mathrm{s}}\left(\boldsymbol{x}-\boldsymbol{x}_{\mathrm{s}}\right)}{4 \pi\left|\boldsymbol{x}-\boldsymbol{x}_{\mathrm{s}}\right|^{3}} \cdot \boldsymbol{n}\right) \mathrm{d} A_{\mathrm{\epsilon}_{\mathrm{s}}} .
$$

At this point we are allowed to choose the $A_{\epsilon_{\mathrm{s}}}$ to be the surfaces of spheres with radii $\epsilon_{\mathrm{s}}$ centred about $\boldsymbol{x}_{\mathrm{s}}$,

$$
\sum_{\mathrm{s}} \int\left(-\frac{m_{\mathrm{s}}}{4 \pi \epsilon_{\mathrm{s}}} \boldsymbol{n}-\boldsymbol{x} \frac{m_{\mathrm{s}}}{4 \pi \epsilon_{\mathrm{s}}^{2}} \boldsymbol{n} \cdot \boldsymbol{n}\right) \mathrm{d} A_{\epsilon_{\mathrm{s}}}=-\sum_{\mathrm{s}} m_{\mathrm{s}} \boldsymbol{x}_{\mathrm{s}} .
$$

Calculate next the dipole contributions with potentials

$$
\phi_{\mathrm{s}}(x)=-\frac{1}{4 \pi} \mu_{\mathrm{s}} \cdot \nabla_{\boldsymbol{x}_{\mathrm{s}}}\left(\frac{1}{\left|x-x_{\mathrm{s}}\right|}\right) .
$$


For any surface $A_{\epsilon}$, however small, it is always possible to choose a $\delta \boldsymbol{x}_{\mathrm{s}}$ such that the potential field at $A_{\epsilon_{\mathrm{s}}}$, associated with a dipole at $\boldsymbol{x}_{\mathrm{s}}$, is a linear superposition of the potential fields due to a source and a sink with strengths of equal magnitude at the points $\boldsymbol{x}_{\mathrm{s}}+\frac{1}{2} \delta \boldsymbol{x}_{\mathrm{s}}$ and $\boldsymbol{x}_{\mathrm{s}}-\frac{1}{2} \delta \boldsymbol{x}_{\mathrm{s}}$

$$
\phi_{\mathrm{s}}(\boldsymbol{x})=\frac{k_{\mathrm{s}}}{4 \pi}\left(-\frac{1}{\left|\boldsymbol{x}-\boldsymbol{x}_{\mathrm{s}}-\frac{1}{2} \boldsymbol{\delta} \boldsymbol{x}_{\mathrm{s}}\right|}+\frac{1}{\left|\boldsymbol{x}-\boldsymbol{x}_{\mathrm{s}}+\frac{1}{2} \boldsymbol{\delta} \boldsymbol{x}_{\mathrm{s}}\right|}\right),
$$

with $k_{s} \delta \boldsymbol{x}_{\mathrm{s}}=\mu_{\mathrm{s}}$. Substituting this expression into the second integral of (3) we have

$$
\begin{aligned}
& \sum_{\mathrm{s}} \int \frac{k_{\mathrm{s}}}{4 \pi}\left\{\left(-\frac{1}{\left|x-x_{\mathrm{s}}-\frac{1}{2} \delta x_{\mathrm{s}}\right|}+\frac{1}{\left|x-x_{\mathrm{s}}+\frac{1}{2} \delta x_{\mathrm{s}}\right|}\right)\right. \\
& \left.-x\left(\frac{x-x_{\mathrm{s}}-\frac{1}{2} \delta x_{\mathrm{s}}}{\left|\boldsymbol{x}-\boldsymbol{x}_{\mathrm{s}}-\frac{1}{2} \delta \boldsymbol{x}_{\mathrm{s}}\right|^{3}}-\frac{\boldsymbol{x}-\boldsymbol{x}_{\mathrm{s}}+\frac{1}{2} \delta x_{\mathrm{s}}}{\left|\boldsymbol{x}-\boldsymbol{x}_{\mathrm{s}}+\frac{1}{2} \delta x_{\mathrm{s}}\right|^{3}}\right) \cdot \boldsymbol{n}\right\} \mathrm{d} A_{\epsilon_{\mathrm{s}}} .
\end{aligned}
$$

Because of the linearity of the integrand the source and the sink contributions may be separated:

$$
\begin{aligned}
& \int \frac{k_{\mathrm{s}}}{4 \pi}\left(-\frac{1}{\left|x-x_{\mathrm{s}}-\frac{1}{2} \delta x_{\mathrm{s}}\right|}-x \frac{\left(x-x_{\mathrm{s}}-\frac{1}{2} \delta x_{\mathrm{s}}\right) \cdot n}{\left|x-x_{\mathrm{s}}-\frac{1}{2} \delta x_{\mathrm{s}}\right|^{3}}\right) \mathrm{d} A_{\epsilon_{\mathrm{s}}} \\
& -\int \frac{k_{\mathrm{s}}}{4 \pi}\left(-\frac{1}{\left|x-x_{\mathrm{s}}+\frac{1}{2} \delta x_{\mathrm{s}}\right|}-x \frac{\left(x-x_{\mathrm{s}}+\frac{1}{2} \delta x_{\mathrm{s}}\right) \cdot n}{\left|x-x_{\mathrm{s}}+\frac{1}{2} \delta x_{\mathrm{s}}\right|^{3}}\right) \mathrm{d} A_{\epsilon_{\mathrm{s}}} .
\end{aligned}
$$

To each integral we next apply Green's reciprocal theorem to transform $A_{\epsilon_{\mathrm{s}}}$ into the surfaces of two spheres with radii $\epsilon_{s}$ and centres in $\boldsymbol{x}_{s}+\frac{1}{2} \delta \boldsymbol{x}_{s}$ and $\boldsymbol{x}_{\mathrm{s}}-\frac{1}{2} \boldsymbol{\delta} \boldsymbol{x}_{\mathrm{s}}$ respectively. With (5) we obtain for the dipole contributions:

$$
-\sum_{\mathrm{s}}\left[k_{\mathrm{s}}\left(\boldsymbol{x}_{\mathrm{s}}+\frac{1}{2} \delta \boldsymbol{x}_{\mathrm{s}}\right)-k_{\mathrm{s}}\left(\boldsymbol{x}_{\mathrm{s}}-\frac{1}{2} \delta \boldsymbol{x}_{\mathrm{s}}\right)\right]=-\sum_{\mathrm{s}} k_{\mathrm{s}} \delta \boldsymbol{x}_{\mathrm{s}}=-\sum_{\mathrm{s}} \mu_{\mathrm{s}} .
$$

The higher-order multipoles can be treated in a similar way. It is evident, however, that these do not contribute to the acceleration reaction.

The final result is

$$
F_{\mathrm{A}}=\rho_{1} \frac{\mathrm{d}}{\mathrm{d} t}\left[v_{\mathrm{c}} \forall-\sum_{\mathrm{s}} M_{q \mathrm{~s}} \frac{\partial^{q}}{\partial x^{q}}(x)_{\mathrm{s}}+\frac{\mathrm{d}}{\mathrm{d} t}\left(x_{\mathrm{c}} \nvdash\right)\right] .
$$

As noted by Landweber and Miloh [2], it is sometimes more convenient to associate the deformation of the body surface with a potential $\phi_{d}$ and use as an alternative to (6):

$$
F_{\mathrm{A}}=\rho_{1} \frac{\mathrm{d}}{\mathrm{d} t}\left[\boldsymbol{v}_{\mathrm{c}} V-\sum_{\mathrm{s}}^{(d)} M_{q \mathrm{~s}} \frac{\partial^{q}}{\partial \boldsymbol{x}^{q}}(x)_{\mathrm{s}}+\int \phi_{\mathrm{d}} n \mathrm{~d} A\right],
$$


where the symbol $\sum_{\mathrm{s}}^{(d)}$ denotes summation over all singularities except those associated with the deformation potential.

\subsection{The steady force}

Rewrite the expression for the force on the body in steady motion as

$$
F_{\mathrm{s}}=\rho_{1} \int\left(\frac{1}{2} u \cdot u n-u u \cdot n\right) \mathrm{d} A+\rho_{1} \int\left(u u \cdot n-u \cdot v_{\mathrm{c}} n-u v_{\mathrm{d}} \cdot \boldsymbol{n}\right) \mathrm{d} A,
$$

or, since $u \cdot n=\left(v_{c}+v_{d}\right) \cdot n$ at the body surface,

$$
F_{\mathrm{s}}=\rho_{1} \int\left(\frac{1}{2} u \cdot u n-u u \cdot n\right) \mathrm{d} A+\rho_{1} \int\left(u v_{\mathrm{c}} \cdot n-u \cdot v_{\mathrm{c}} n\right) \mathrm{d} A
$$

With the aid of the relation $u v_{c} \cdot n-u \cdot v_{c} n=v_{c} \times(u \times n)$ we obtain for the second integral in (8)

$$
\int v_{\mathrm{c}} \times(\boldsymbol{u} \times \boldsymbol{n}) \mathrm{d} A=-\boldsymbol{v}_{\mathrm{c}} \times \int(\nabla \times u) \mathrm{d} V=0 .
$$

Applying the divergence theorem to the volume bounded externally by the body surface and internally by surfaces $A_{\epsilon_{\mathrm{s}}}$ enclosing small regions about the points $\boldsymbol{x}_{\mathrm{s}}$, we get

$$
F_{\mathrm{s}}=\rho_{1} \int\left(\frac{1}{2} u \cdot u n-u u \cdot n\right) \mathrm{d} A=\rho_{1} \sum_{\mathrm{s}} \int\left(\frac{1}{2} u \cdot u n-u u \cdot n\right) \mathrm{d} A_{\epsilon_{\mathrm{s}}} \cdot
$$

Again, at each singular point the velocity field can be assumed to consist of a regular and a singular part of the form (4), hence

$$
\begin{aligned}
& \boldsymbol{F}_{\mathrm{s}}=\rho_{1} \sum_{\mathrm{s}}\left\{\left(\frac{1}{2} \boldsymbol{u}_{\mathrm{r}} \cdot \boldsymbol{u}_{\mathrm{r}} n-u_{\mathrm{r}} \boldsymbol{u}_{\mathrm{r}} \cdot \boldsymbol{n}\right) \mathrm{d} A_{\epsilon_{\mathrm{s}}}+\rho_{1} \sum_{\mathrm{s}} \int\left(\frac{1}{2} \boldsymbol{u}_{\mathrm{s}} \cdot \boldsymbol{u}_{\mathrm{s}} n-u_{\mathrm{s}} \boldsymbol{u}_{\mathrm{s}} \cdot \boldsymbol{n}\right) \mathrm{d} A_{\mathrm{\epsilon}_{\mathrm{s}}}\right. \\
& +\rho_{1} \sum_{\mathrm{s}} \int\left(\boldsymbol{u}_{\mathrm{s}} \cdot \boldsymbol{u}_{\mathrm{r}} n-u_{\mathrm{r}} u_{\mathrm{s}} \cdot n-u_{\mathrm{s}} u_{\mathrm{r}} \cdot n\right) \mathrm{d} A_{\epsilon_{\mathrm{s}}} \cdot
\end{aligned}
$$

For the first integral we obtain

$$
\lim _{A_{\epsilon_{\mathrm{s}}} \rightarrow 0} \int\left(\frac{1}{2} \boldsymbol{u}_{\mathrm{r}} \cdot \boldsymbol{u}_{\mathrm{r}} \boldsymbol{n}-\boldsymbol{u}_{\mathrm{r}} \boldsymbol{u}_{\mathrm{r}} \cdot \boldsymbol{n}\right) \mathrm{d} A_{\epsilon_{\mathrm{s}}}=0
$$

For the second integral we can calculate the contribution of each singularity separately by choosing a surface $S_{\mathrm{s}}$ about the point $\boldsymbol{x}_{\mathrm{s}}$ in question and by applying next Gauss' theorem to the volumes $V_{\mathrm{s}}$ bounded by the surfaces $S_{\mathrm{s}}$ and $A_{\text {es }}$,

$$
\begin{aligned}
\int\left(\frac{1}{2} u_{\mathrm{s}} \cdot u_{\mathrm{s}} n-u_{\mathrm{s}} u_{\mathrm{s}} \cdot n\right) \mathrm{d} A_{\epsilon_{\mathrm{s}}}= & \int\left(\frac{1}{2} u_{\mathrm{s}} \cdot u_{\mathrm{s}} n-u_{\mathrm{s}} u_{\mathrm{s}} \cdot n\right) \mathrm{d} S_{\mathrm{s}} \\
& -\int\left(\frac{1}{2} \nabla_{x_{\mathrm{s}}}\left(u_{\mathrm{s}} \cdot u_{\mathrm{s}}\right)-\nabla_{x_{\mathrm{s}}} \cdot\left(u_{\mathrm{s}} u_{\mathrm{s}}\right)\right) \mathrm{d} V_{\mathrm{s}} .
\end{aligned}
$$


Because the singular velocity fields are both irrotational and solenoidal in $V_{\mathrm{s}}$ the volume integral equals zero. And since $\left|\boldsymbol{u}_{\mathrm{s}}\right|$ is at least as small as $\left|\boldsymbol{x}-\boldsymbol{x}_{\mathrm{s}}\right|^{-3}$, the choice of $S_{\mathrm{s}}$ as a sphere of infinitely large radius shows the integral over $S_{\mathrm{s}}$ to vanish as well. To calculate the third integral, consider first the contribution of the monopoles:

$$
\sum_{\mathrm{s}} \int\left[\frac{m_{\mathrm{s}}\left(x-x_{\mathrm{s}}\right)}{4 \pi\left|x-x_{\mathrm{s}}\right|^{3}} \cdot u_{\mathrm{r}} n-u_{\mathrm{r}} \frac{m_{\mathrm{s}}\left(x-x_{\mathrm{s}}\right)}{4 \pi\left|x-x_{\mathrm{s}}\right|^{3}} \cdot n-\frac{m_{\mathrm{s}}\left(x-x_{\mathrm{s}}\right)}{4 \pi\left|x-x_{\mathrm{s}}\right|^{3}} u_{\mathrm{r}} \cdot n\right] \mathrm{d} A_{\epsilon_{\mathrm{s}}},
$$

which, by choosing the $A_{\mathrm{t}_{\mathrm{s}}}$ to be surfaces of spheres with radii $\epsilon_{\mathrm{s}}$ and centres in $\boldsymbol{x}_{\mathrm{s}}$, equals

$$
\begin{array}{r}
\sum_{\mathrm{s}} \frac{m_{\mathrm{s}}}{4 \pi \epsilon_{\mathrm{s}}^{2}} \int\left(\boldsymbol{n} \cdot \boldsymbol{u}_{\mathrm{r}} \boldsymbol{n}-\boldsymbol{u}_{\mathrm{r}} \boldsymbol{n} \cdot \boldsymbol{n}-\boldsymbol{n} \boldsymbol{u}_{\mathrm{r}} \cdot \boldsymbol{n}\right) \mathrm{d} A_{\epsilon_{\mathrm{s}}} \\
=-\sum_{\mathrm{s}} \frac{m_{\mathrm{s}}}{4 \pi \epsilon^{2}} \int \boldsymbol{u}_{\mathrm{r}} \mathrm{d} A_{\epsilon_{\mathrm{s}}}=-\sum_{\mathrm{s}} m_{\mathrm{s}} \boldsymbol{u}_{\mathrm{r}}\left(\boldsymbol{x}_{\mathrm{s}}\right) .
\end{array}
$$

The dipole contributions can be obtained from this result in a way which resembles the calculation of these contributions to the acceleration reaction. For any surface $A_{\mathrm{f}_{\mathrm{g}}}$, however small, a $\delta x_{\mathrm{s}}$ can be chosen such that the velocity field at $A_{\epsilon_{\mathrm{s}}}$ is a superposition of the velocity fields of a source and a sink at $x_{s}+\frac{1}{2} \delta x_{s}$ and $x_{s}-\frac{1}{2} \delta x_{s}$ respectively,

$$
u_{\mathrm{s}}(x)=\frac{k_{\mathrm{s}}}{4 \pi}\left(\frac{x-x_{\mathrm{s}}-\frac{1}{2} \delta x_{\mathrm{s}}}{\left|x-x_{\mathrm{s}}-\frac{1}{2} \delta x_{\mathrm{s}}\right|^{3}}-\frac{x-x_{\mathrm{s}}+\frac{1}{2} \delta x_{\mathrm{s}}}{\left|x-x_{\mathrm{s}}+\frac{1}{2} \delta x_{\mathrm{s}}\right|^{3}}\right),
$$

with $k_{s} \delta x_{\mathrm{s}}=\mu_{\mathrm{s}}$. The source and the sink contributions can be considered separately, whence we obtain

$$
\begin{aligned}
& \frac{k_{\mathrm{s}}}{4 \pi} \int\left[\frac{\left(x-x_{\mathrm{s}}-\frac{1}{2} \delta x_{\mathrm{s}}\right)}{\left|x-x_{\mathrm{s}}-\frac{1}{2} \delta x_{\mathrm{s}}\right|^{3}} \cdot u_{\mathrm{r}} n-u_{\mathrm{r}} \frac{\left(x-x_{\mathrm{s}}-\frac{1}{2} \delta x_{\mathrm{s}}\right)}{\left|x-x_{\mathrm{s}}-\frac{1}{2} \delta x_{\mathrm{s}}\right|^{3}} \cdot n-\frac{\left(x-x_{\mathrm{s}}-\frac{1}{2} \delta s_{\mathrm{s}}\right)}{\left|x-x_{\mathrm{s}}-\frac{1}{2} \delta x_{\mathrm{s}}\right|^{3}} u_{\mathrm{r}} \cdot n\right] \mathrm{d} A_{\mathrm{\epsilon}_{\mathrm{s}}} \\
& -\frac{k_{\mathrm{s}}}{4 \pi} \int\left[\frac{\left(x-x_{\mathrm{s}}+\frac{1}{2} \delta x_{\mathrm{s}}\right)}{\left|x-x_{\mathrm{s}}+\frac{1}{2} \delta x_{\mathrm{s}}\right|^{3}} \cdot u_{\mathrm{r}} n-u_{\mathrm{r}} \frac{\left(x-x_{\mathrm{s}}+\frac{1}{2} \delta x_{\mathrm{s}}\right)}{\left|x-x_{\mathrm{s}}+\frac{1}{2} \delta x_{\mathrm{s}}\right|^{3}} \cdot n\right. \\
& \left.-\frac{\left(x-x_{\mathrm{s}}+\frac{1}{2} \delta x_{\mathrm{s}}\right)}{\left|x-x_{\mathrm{s}}+\frac{1}{2} \delta x_{\mathrm{s}}\right|^{3}} u_{\mathrm{r}} \cdot n\right] \mathrm{d} A_{\mathrm{c}_{\mathrm{s}}} .
\end{aligned}
$$

Next, apply the divergence theorem to each integral in order to transform the $A_{\epsilon}$ into surfaces of spheres with radii $\epsilon_{\mathrm{s}}$ and centres at $\boldsymbol{x}_{\mathrm{s}}+\frac{1}{2} \delta \boldsymbol{x}_{\mathrm{s}}$ and $\boldsymbol{x}_{\mathrm{s}}-\frac{1}{2} \delta \boldsymbol{x}_{\mathrm{s}}$. With the monopole result (9), this yields

$$
\begin{aligned}
-\sum_{\mathrm{s}}\left[k_{\mathrm{s}} \boldsymbol{u}_{\mathrm{r}}\left(\boldsymbol{x}_{\mathrm{s}}+\frac{1}{2} \delta \boldsymbol{x}_{\mathrm{s}}\right)-k_{\mathrm{s}} \boldsymbol{u}_{\mathrm{r}}\left(\boldsymbol{x}_{\mathrm{s}}-\frac{1}{2} \delta \boldsymbol{x}_{\mathrm{s}}\right)\right] & =-\sum_{\mathrm{s}} k_{\mathrm{s}} \delta \boldsymbol{x}_{\mathrm{s}} \cdot \nabla_{\boldsymbol{x}}\left(\boldsymbol{u}_{\mathrm{r}}\right)_{\mathrm{s}} \\
& =-\sum_{\mathrm{s}} \mu_{\mathrm{s}} \cdot \nabla_{x}\left(\boldsymbol{u}_{\mathrm{r}}\right)_{\mathrm{s}} .
\end{aligned}
$$


The contribution of the higher-order poles can be found in a similar way, and we finally have for the steady force

$$
F_{\mathrm{s}}=-\rho_{1} \sum_{\mathrm{s}} M_{q \mathrm{~s}} \frac{\partial^{q}}{\partial \boldsymbol{x}^{q}}\left(\boldsymbol{u}_{\mathrm{r}}\right)_{\mathrm{s}}
$$

From (7) and (10) the generalized Lagally theorem is obtained:

$$
F=\rho_{1} \frac{\mathrm{d}}{\mathrm{d} t}\left[v_{\mathrm{c}} \forall-\sum_{\mathrm{s}}^{(d)} M_{q \mathrm{~s}} \frac{\partial^{q}}{\partial x^{q}}(x)_{\mathrm{s}}+\int \phi_{\mathrm{d}} n \mathrm{~d} A\right]-\rho_{1} \sum_{\mathrm{s}} M_{q \mathrm{~s}} \frac{\partial^{q}}{\partial x^{q}}\left(u_{\mathrm{r}}\right)_{\mathrm{s}} .
$$

\section{Examples}

Consider a sphere with radius $a(t)$ and centre at $x_{\mathrm{c}}$, moving with velocity $v$ in an unbounded, nonuniform and unsteady potential flow, which has potential $\phi\left(x_{\mathrm{c}}, t\right)$ and velocity $u\left(x_{\mathrm{c}}, t\right)$ in $\boldsymbol{x}_{\mathrm{c}}$ in the absence of the sphere. An approximation to the potential field on the surface of the sphere is obtained by representing the sphere by a source and a dipole at the centre, with a dipole strength associated with the velocity in $\boldsymbol{x}_{\mathrm{c}}$ in the absence of the sphere,

$$
\begin{aligned}
\phi(x, t)= & u\left(x_{\mathrm{c}}, t\right) \cdot\left(x-x_{\mathrm{c}}\right)-\frac{a^{2} \mathrm{~d} a / \mathrm{d} t}{\left|x-x_{\mathrm{c}}\right|} \\
& -\frac{1}{2} a^{3}\left(u\left(x_{\mathrm{c}}, t\right)-v\right) \cdot \nabla_{x} \frac{1}{\left|x-x_{\mathrm{c}}\right|}+O\left(\left|x-x_{\mathrm{c}}\right|^{-5}\right):
\end{aligned}
$$

The generalized Lagally theorem then yields as an approximation to the force on the body:

$$
\begin{aligned}
F & \simeq \rho_{1}\left\{\frac{\mathrm{d}}{\mathrm{d} t}\left[\frac{4}{3} \pi a^{3} v+2 \pi a^{3}\left(u\left(x_{\mathrm{c}}, t\right)-v\right)\right]\right. \\
& \left.-4 \pi a^{2} \frac{\mathrm{d} a}{\mathrm{~d} t} u\left(x_{\mathrm{c}}, t\right)+2 \pi a^{3}\left(u\left(x_{\mathrm{c}}, t\right)-v\right) \cdot \nabla_{x}(u)_{x_{\mathrm{c}}}\right\} .
\end{aligned}
$$

The first term on the right-hand side is the acceleration reaction. Since only monopoles and dipoles can contribute to this term and since there are no bodies or fixed surfaces present in the fluid in the case considered here, a full description of the flow field would yield exactly the same result as given in (11). This implies that the acceleration reaction of a rigid sphere in an impulsively generated unbounded inhomogeneous potential flow follows directly from the value of this potential field at the centre of the sphere. For a massless sphere we have

$$
v=3 u\left(x_{\mathrm{c}}\right)
$$


a result derived earlier by van Wijngaarden [5].

The relation (11) can also be written as

$$
\boldsymbol{F} \simeq \rho_{1}\left\{2 \pi a^{3}\left(\frac{\mathrm{D} u}{\mathrm{D} t}\right)_{x_{\mathrm{c}}}-\frac{2}{3} \pi a^{3} \frac{\mathrm{d} v}{\mathrm{~d} t}+2 \pi a^{2} \frac{\mathrm{d} a}{\mathrm{~d} t}\left(\boldsymbol{u}\left(x_{\mathrm{c}}, t\right)-v\right)\right\},
$$

where $\mathrm{D} / \mathrm{D} t=\partial / \partial t+\boldsymbol{u} \cdot \nabla_{\boldsymbol{x}}$, the material derivative following the fluid and which is to be evaluated at the centre of the sphere.

An important special case is that of a rigid body (for convenience taken to be a sphere) moving in an unbounded inhomogeneous unsteady potential flow, where the length scale of variations of the external flow field considerably exceeds the body's dimensions. The velocity field in the absence of the sphere can then be approximated by

$$
u(x, t)=u_{0}(t)+x \cdot\left(\nabla_{x} u\right)_{0}(t) .
$$

When a sphere is placed at $\boldsymbol{x}_{\mathrm{c}}$ this results in a potential field which can be represented by the original potential field and contributions from a dipole and a quadrupole both placed at the centre of the sphere. Only the dipole contributes to the acceleration reaction,

$$
\rho_{1} \frac{\mathrm{d}}{\mathrm{d} t}\left[\frac{4}{3} \pi a^{3} v+2 \pi a^{3}\left(u\left(x_{c}, t\right)-v\right)\right]
$$

The contribution from the quadrupole to the steady force has the form of a second-order derivative of the regular flow field, to be evaluated at $\boldsymbol{x}_{\mathrm{c}}$. Because of the linearity of this flow field the contribution is identically zero. Together with the dipole contribution

$$
2 \pi a^{3}\left(u\left(x_{\mathrm{c}}, t\right)-v\right) \cdot \nabla_{x}(u)_{x_{\mathrm{c}}}
$$

the exact force can be written as

$$
F=\rho_{1}\left\{2 \pi a^{3}\left(\frac{\mathrm{D} u}{\mathrm{D} t}\right)_{x_{\mathrm{c}}}-\frac{2}{3} \pi a^{3} \frac{\mathrm{d} v}{\mathrm{~d} t}\right\}
$$

The relations (12) and (13) which can also be inferred from work by G.I. Taylor (see e.g. Taylor [6]), are associated in recent gas-liquid two-phase flow literature with the names of Voinov [7] and Yakimov [8], and are considered to be the correct equations describing the average relative motion of the gas phase when viscosity effects are neglected.

When the average gas velocity and the average liquid velocity are denoted by $U_{\mathrm{g}}$ and $U_{1}$ respectively, and the material derivatives with respect to the averaged velocities of the phases are given by

$$
\frac{\mathrm{D}}{\mathrm{D} t}=\frac{\partial}{\partial t}+U_{1} \cdot \nabla, \frac{\mathrm{d}}{\mathrm{d} t}=\frac{\partial}{\partial t}+U_{\mathrm{g}} \cdot \nabla
$$

it is believed that the Voinov-equation (12) suggests that the equation of motion for the gas bubbles in a bubbly liquid is of the form

$$
\frac{\mathrm{d}}{\mathrm{d} t} m U_{\mathrm{g}}-\frac{\mathrm{D}}{\mathrm{D} t} m U_{1}=\rho_{1} K \frac{\mathrm{D}}{\mathrm{D} t} U_{1}
$$


where $V$ is the average bubble volume and $m$ is the average added mass which may be a function of the volumetric gas fraction. Although the Lagally theorem shows the Voinovequation to describe the motion of one single bubble in an inhomogeneous flow field correctly, there is no justification for a direct extension of it in the form (14) as a description of the averaged motion of a swarm of bubbles. An averaging proces might for instance reveal that flow-field variations over a length scale relevant for the motion of the individual bubbles vanish in a continuum description of the flow in terms of averaged flow parameters and their derivatives with respect to 'macroscopic' length scales. The results of such an averaging process have recently been given by Biesheuvel \& van Wijngaarden [9].

\section{Acknowledgement}

I am grateful to Professor L. van Wijngaarden for his stimulating discussions and comments. I would also like to thank the F.O.M. foundation for financial support, under contract no. TTN 22.0213.

\section{References}

[1] L. Landweber and C.S. Yih, Forces, moments and added masses for Rankine bodies. J. Fluid Mech. 1 (1956) 319-336.

[2] L. Landweber and T. Miloh, Unsteady Lagally theorem for multipoles and deformable bodies. J. Fluid Mech. 96 (1980) 33-46.

[3] L. Landweber and T. Miloh, Corrigendum to "Unsteady Lagally theorem for multipoles and deformable bodies". J. Fluid Mech. 112 (1981) 502.

[4] L. van Wijngaarden, On the motion of gas bubbles in a perfect liquid. Arch. Mech. 34 (1982) 343-349.

[5] L. van Wijngaarden, Hydrodynamic interaction between gas bubbles in liquid. J. Fluid Mech. 77 (1976) 27-44.

[6] G.I. Taylor, The forces on a body in a curved or converging stream of fluid. Proc. R. Soc. Lond. A70 (1928) 260-283.

[7] O.V. Voinov, Force acting on a sphere in an inhomogeneous flow of an incompressible fluid. J. Appl. Mech. Tech. Phys. 14 (1973) 592-594.

[8] Yu.L. Yakimov, Forces acting on a small body in a flowing incompressible liquid and equations of motion of a two-phase medium. Fluid Dynamics 8 (1973) 411-418.

[9] A. Biesheuvel and L. van Wijngaarden, Two-phase flow equations for a dilute dispersion of gas bubbles in liquid. J. Fluid Mech. 148 (1984) 301-318. 